\title{
Hamiltonian analysis of a topological theory in the presence of boundaries
}

\author{
Alejandro Corichi ${ }^{1, \text {, }}$ and Tatjana Vukašinac ${ }^{2}$, \\ ${ }^{1}$ Centro de Ciencias Matemáticas, Universidad Nacional Autónoma de México, \\ UNAM-Campus Morelia, A. Postal 61-3, Morelia, Michoacán 58090, Mexico \\ ${ }^{2}$ Facultad de Ingeniería Civil, Universidad Michoacana de San Nicolás de Hidalgo, Morelia, Michoacán 58000, Mexico
}

\begin{abstract}
We perform the canonical Hamiltonian analysis of a topological gauge theory, that can be seen both as a theory defined on a four dimensional spacetime region with boundaries - the bulk theory-, or as a theory defined on the boundary of the region -the boundary theory-. In our case the bulk theory is given by the 4-dimensional $U(1)$ Pontryagin action and the boundary one is defined by the $U(1)$ Chern-Simons action. We analyse the conditions that need to be imposed on the bulk theory so that the total Hamiltonian, smeared constraints and generators of gauge transformations be well defined (differentiable) for generic boundary conditions. We pay special attention to the interplay between the constraints and boundary conditions in the bulk theory on the one side, and the constraints in the boundary theory, on the other side. We illustrate how both theories are equivalent, despite the different canonical variables and constraint structure, by explicitly showing that they both have the same symmetries, degrees of freedom and observables.
\end{abstract}

PACS numbers: 03.50.Kk, 11.15.Yc, 11.10.Ef

\section{INTRODUCTION}

It is well known that gauge theories defined on spacetime regions with boundary can have degrees of freedom and observables localized on the boundary (see for example [1]). Among them are topological theories that generate effective theories on the boundary. There is an extensive work on 3-dimensional Chern-Simons theory that generates a Wess-Zumino-Witten theory on the boundary [2 12]. Another example is a 5-dimensional Abelian Chern-Simons theory to which it corresponds an effective 4-dimensional theory on the boundary [13, 14]. Yet another example is a 4-dimensional BF theory in a bounded region whose surface observables were studied in [8, 15, 16]. In all of these cases the bulk theory is topological, in the sense that it does not have any local degrees of freedom, but there are degrees of freedom on the boundary. Even in the case of non-Abelian higher dimensional Chern-Simons theories (that are not topological) or 4-dimensional gravity in spacetimes with boundaries, there are induced degrees of freedom on the boundary [15, 17]. Here we will consider the simplest case when the bulk theory and the boundary one are straightforwardly related, namely when the Lagrangian density of the bulk theory is a total derivative.

One well known fact about topological theories of some kind, namely when the Lagrangian density can be written as a total derivative -and can thus be integrated by parts into a surface integral-, is that they are "equivalent" to that theory on the boundary. While there is a clear equivalence as suggested by the equality of their actions, one can still ask how, and in which sense are the two theories equivalent. Note that this is a very old question that can be put in the category of a "classical holographic principle" (to distinguish it from the modern holographic principle widely studied in the literature). There is, of course, an ample literature on the subject (see for instance [8] for a recent review, and references therein). An analysis of the covariant formulation of such topological theories has been performed before and it is rather easy to see that, in the covariant Hamiltonian formalism (where the phase space is defined by the solutions to the classical equations of motion), the theory on the bulk reduces to that on the boundary in a rather natural manner; the symplectic structure and therefore any Hamiltonian observable of the bulk theory only have contributions from the fields at the boundary (see for instance [18] for an overview). There is no contribution from the bulk in the relevant integrals.

The natural question that arises then is how to compare the two theories from the canonical Hamiltonian perspective. It is easy to see that there exists a potential mismatch even in the definition of the fundamental variables of the theory. While the "configuration variables" on the boundary might be naturally defined by the evaluation of the corresponding bulk variables on the boundary, the same is not true of the canonically defined momenta. Even more, the constraints and their corresponding algebra might be different: Are they first/second class? Do they generate the same gauge symmetries? If the two theories under consideration posses these distinct features, one might then ask in which sense

*Electronic address: corichi@matmor.unam.mx

$\dagger$ Electronic address: tatjana@umich.mx 
are the two theories equivalent. The purpose of this manuscript is to consider all these issues for the simplest of such theories, namely the $4 D U(1)$ Pontryagin action on the bulk region and the corresponding boundary theory defined by a $U(1)$ Chern-Simons action. As we shall see in detail, the canonical analysis of this theory, viewed as a bulk theory or a boundary one, shows that the structure and algebra of constraints are different in these two approaches. Thus it becomes a pressing question how to compare these two theories. To offer such comparison is the objective of this manuscript.

Apart from the differences we have mentioned between the two theories, there is another important issue that needs to be addressed. When one is considering a theory with boundaries, it is fundamental to consider boundary conditions for the dynamical variables. These conditions might me physically motivated, or can be chosen to render the theory self-consistent. In the covariant approach this entails to having a well defined action principle, which means the action should be differentiable (see, for instance [8, 18]). If the original action is not differentiable with the chosen boundary conditions, one might need to supplement it with a boundary term. In the canonical Hamiltonian description, one adopts a slightly modified viewpoint. One starts with the action and through the standard Dirac procedure one obtains canonical momenta, possible primary constraints and a Hamiltonian. Then one imposes that all relevant quantities be differentiable. This condition might require the introduction of boundary conditions, extra boundary terms, or both (see for instance [19] and [8] for a review). On the other hand the corresponding boundary theory is defined on a region without a boundary (the boundary of the boundary is zero), so there are not such issues arising in its canonical formulation.

Here we shall explore the relation between the two approaches, bulk and boundary, and show that there is an interplay between the boundary conditions that need to be imposed in the bulk theory in order to be well defined in the presence of boundaries, and the constraints in the induced boundary theory. This result can be seen as an indication that the boundary conditions could be treated as second class constraints in the phase space (as explored, for instance, in [20 22]). A purely geometrical approach to the analysis of gauge theories in spacetimes with boundary has been developed in [23, 24].

As we shall show in detail, one can see in a clear fashion that the two theories are equivalent in a precise sense. This will involve studying in detail the physical degrees of freedom of the theories, through their physical observables, and the generators of interesting gauge symmetries such as internal gauge and spacetime diffeomorphisms. The canonical analysis of the Pontryagin and the Chern-Simons theories is not new, of course. For instance, it has been performed in [25], and some of their results are closely related to ours. There are, however, important technical and conceptual differences, given that our approach to comparing both theories is different. We shall elaborate in sections below. The extension to a non-Abelian gauge group is straightforward and does not shed any new insights. For simplicity and in order to focus on the conceptual issues we shall only consider the Abelian theory.

The structure of the paper is as follows. In Section $\amalg$ we present the Hamiltonian analysis of the Pontryagin theory on a 4-dimensional spacetime region with boundary. We show that there are three equivalent canonical Hamiltonians and analyse the boundary conditions that one needs to impose in order to have a well defined total Hamiltonian. We also construct gauge generators and a set of observables for this theory. In Section III we perform the canonical decomposition of the Chern-Simons theory defined on the 3-dimensional "time-like" boundary, and also construct the corresponding gauge generators and a set of observables. In Section. IV] we summarize the results and show the relation between canonical descriptions of the two theories. An outlook is the subject of Sec. $\mathrm{V}$.

In this manuscript we adopt the abstract index notation of Penrose, where geometrical objects are equipped with an abstract index that does not "take any values". Thus, while for instance a connection $A_{a}$ on spacetime and its pullback to the boundary will be denoted by the same symbol $A_{a}$, it should be clear, we hope, that the two objects are defined on different manifolds. We shall not use a new symbol to denote pullbacks or restrictions for notational simplicity, and hope the meaning shall become clear from the context. We shall use a fully covariant approach in the canonical decomposition, without fixing a foliation nor a coordinate system. We have kept the mathematical sophistication to the level of the theoretical physics literature, without the rigour of mathematical physics employed, for instance, in [24].

\section{HAMILTONIAN ANALYSIS OF THE PONTRYAGIN THEORY}

In this section we perform the canonical Hamiltonian analysis of the theory in the bulk, namely of the Pontryagin theory on the four dimensional spacetime region $\mathcal{M}$, with boundary, which we shall take to be of the form $\mathcal{M}=I \times \Sigma$, with $I$ a closed interval. $\Sigma$ is a three dimensional manifold with boundary $\partial \Sigma$ that we shall take to have the topology of a two sphere $S^{2}$. This section has three parts. In the first one, we perform the canonical $3+1$ decomposition of the theory, following the Dirac algorithm. In the second part we construct physical observables out of simple expressions and in the third part we construct the generators of gauge symmetries. 


\section{A. Canonical decomposition}

The Pontryagin action for the Abelian theory is

$$
S_{\mathrm{P}}=\int_{\mathcal{M}} \mathbf{F} \wedge \mathbf{F}=\frac{1}{4} \int_{\mathcal{M}} \mathrm{d}^{4} x \tilde{\varepsilon}^{a b c d} F_{a b} F_{c d}
$$

where $\mathbf{F}=\mathrm{d} \mathbf{A}$ is the field strength two-form, the curvature of the $U(1)$ connection one-form $\mathbf{A}$ and $\tilde{\varepsilon}^{a b c d}$ is the Levi-Civita tensor density. Here we have set the dimension-full parameter $\theta=1$, since there is no loss of generality as we are not coupling the fundamental variables to another field. We introduce a foliation of the spacetime $\mathcal{M}=I \times \Sigma$ and a "time" function $t$ such that hypersurfaces $\Sigma_{t}$, that correspond to $t=$ const, are diffeomorphic to $\Sigma$. Then we can introduce a time evolution vector field $t^{a}:=\left(\frac{\partial}{\partial t}\right)^{a}$ such that $t^{a} \partial_{a} t=1$. Using the identity $\tilde{\varepsilon}^{a b c d}=4 t^{[a} \tilde{\varepsilon}^{b c d]}($ that implies $\tilde{\varepsilon}^{a b c d} t_{a}=\tilde{\varepsilon}^{b c d}$, where $\left.t_{a}:=\partial_{a} t\right)$, the action can be expressed as

$$
S_{\mathrm{P}}=\int_{\mathcal{M}} \mathrm{d}^{4} x \tilde{\varepsilon}^{a b c}\left(t^{d} F_{d a}\right) F_{b c}=\int_{\mathcal{M}} \mathrm{d}^{4} x \tilde{\varepsilon}^{a b c} F_{b c}\left(£_{t} A_{a}-\partial_{a} \phi\right)
$$

where $\phi:=t \cdot \mathbf{A}$ and we have used the Cartan identity $£_{t} A_{a}=t^{b} F_{b a}+\partial_{a}(t \cdot \mathbf{A})$. Then, the canonical momenta is given by

$$
\tilde{\Pi}^{a}:=\frac{\delta \mathcal{L}}{\delta\left(£_{t} A_{a}\right)}=\tilde{\varepsilon}^{a b c} F_{b c}
$$

where $\mathcal{L}$ is the Lagrangian density. It should be noted that the momenta are intrinsic to $\Sigma$, since $t_{a} \tilde{\Pi}^{a}=0$. The kinematical phase space is 8 -dimensional (per point) and is parametrized by the set of canonical variables $\left(\phi, \tilde{\Pi}_{\phi} ; A_{a}, \tilde{\Pi}^{a}\right)$ where $\tilde{\Pi}_{\phi}:=t \cdot \tilde{\boldsymbol{\Pi}}$ and $A_{a}$ is the pullback of $\mathbf{A}$ to $\Sigma$.

The theory has four primary constraints

$$
\begin{aligned}
\tilde{\mathcal{C}} & :=\tilde{\Pi}_{\phi} \approx 0, \\
\tilde{\mathcal{C}}^{a} & :=\tilde{\Pi}^{a}-\tilde{\varepsilon}^{a b c} F_{b c} \approx 0 .
\end{aligned}
$$

Note that $t_{a} \mathcal{C}^{a}=0$, so $\mathcal{C}^{a}$ is intrinsic to $\Sigma$.

The canonical Hamiltonian is defined through the Legendre transformation,

$$
H_{\mathrm{C}}=\int_{\Sigma} \mathrm{d}^{3} x\left[\left(£_{t} \phi\right) \tilde{\Pi}_{\phi}+\left(£_{t} A_{a}\right) \tilde{\Pi}^{a}-\mathcal{L}\right]=\int_{\Sigma} \mathrm{d}^{3} x\left[\left(£_{t} \phi\right) \tilde{\Pi}_{\phi}+\left(£_{t} A_{a}\right) \tilde{\Pi}^{a}-\tilde{\varepsilon}^{a b c} F_{b c}\left(£_{t} A_{a}-\partial_{a} \phi\right)\right] .
$$

Since the Lagrangian is linear in $£_{t} A_{a}$, this velocity term cannot be expressed as a function of $\tilde{\Pi}^{a}$. As a result we can write the canonical Hamiltonian in several different forms that we are going to explore in detail in the following sections.

The general strategy for the canonical analysis of the constrained system will be the following. We are going to assume that the Lagrangian theory is well defined, without paying attention to the boundary conditions that might have appeared there. Instead, we shall proceed with the canonical theory anew: we start with generic boundary conditions, and let the theory "tell" us what modifications, in terms of boundary conditions or extra terms, might be needed. The first such conditions might come from the first step, namely in having a well defined, differentiable, canonical Hamiltonian. That is the subject of the sections that follow.

Let us end this part with a comment. In some part of the literature, one can find that certain boundary conditions are imposed in the covariant action principle, in order to make it differentiable. These conditions are then "carried over" to the canonical analysis and could yield, consequently, a different sector of the theory. Our viewpoint here is to impose any consistency conditions only at the canonical level, and consider the most general variations that are allowed.

\section{First approach}

Let us start by considering the first form that the canonical Hamiltonian can take. If we substitute $\tilde{\varepsilon}^{a b c} F_{b c}=\tilde{\Pi}^{a}$ and $\tilde{\Pi}_{\phi}=0$ in (6), the canonical Hamiltonian becomes

$$
H_{\mathrm{C} 1}=\int_{\Sigma} \mathrm{d}^{3} x \tilde{\Pi}^{a} \partial_{a} \phi .
$$


The variation of $H_{\mathrm{C} 1}$ on the phase space is then

$$
\delta H_{\mathrm{C} 1}=\int_{\Sigma} \mathrm{d}^{3} x\left[\left(\partial_{a} \phi\right) \delta \tilde{\Pi}^{a}-\left(\nabla_{a} \tilde{\Pi}^{a}\right) \delta \phi\right]+\int_{\Sigma} \mathrm{d}^{3} x \nabla_{a}\left(\tilde{\Pi}^{a} \delta \phi\right) .
$$

Note that since $\tilde{\Pi}^{a}$ is a vector density of weight $1, \nabla_{a} \tilde{\Pi}^{a}=\partial_{a} \tilde{\Pi}^{a 1}$. Now, we can use Stokes' theorem for vector densities of weight 1 , so that

$$
\int_{\Sigma} \mathrm{d}^{3} x \nabla_{a}\left(\tilde{\Pi}^{a} \delta \phi\right)=\int_{\partial \Sigma} \mathrm{d} S_{a} \tilde{\Pi}^{a} \delta \phi
$$

where $\mathrm{d} S_{a}=\frac{1}{2} \tilde{\varepsilon}_{a b c} \mathrm{~d} x^{b} \wedge \mathrm{d} x^{c}$. As a result, $H_{\mathrm{C} 1}$ is a differentiable function if this boundary term vanishes for arbitrary variations $\delta \phi$. Let us introduce the coordinate $r$ such that $\partial \Sigma$ is defined as the surface $r=$ const. and $r_{a}=\partial_{a} r$ is its normal 1-form. Then, $\mathrm{d} S_{a} \sim r_{a}$ and the condition for differentiability takes the form

$$
\left.\left.r_{a} \tilde{\Pi}^{a}\right|_{\partial \Sigma} \approx r_{a} \tilde{\varepsilon}^{a b c} F_{b c}\right|_{\partial \Sigma}=\left.\tilde{\varepsilon}^{b c} F_{b c}\right|_{\partial \Sigma}=0 .
$$

This implies that the pullback of the curvature to the boundary vanishes: $\left.F_{a b}\right|_{\partial \Sigma}=0$. We shall demand that these conditions hold on the whole boundary $I \times \partial \Sigma$. As we stated earlier, we are assuming that the boundary of $\Sigma$, has the topology of a two sphere, $\partial \Sigma \approx S^{2}$. This in turn implies that $H^{1}(\partial \Sigma)=0$, so the only allowed variations of the pullback of $\mathbf{A}$ to the boundary are of the form, $\delta A_{a}=\left.\partial_{a} \lambda\right|_{\partial \Sigma}$, where $\lambda$ is an arbitrary function on $\partial \Sigma$. Had we allowed for more complicated topologies (like a two torus) or punctures, we would have had more possibilities (and boundary degrees of freedom).

It should be noted that in requiring that the canonical Hamiltonian be differentiable, one has to impose boundary conditions on the connection, restricting to those that are flat on $\partial \Sigma$.

The total Hamiltonian is:

$$
H_{\mathrm{T} 1}=\int_{\Sigma} \mathrm{d}^{3} x\left(\tilde{\Pi}^{a} \partial_{a} \phi+u \tilde{\mathcal{C}}+u_{a} \tilde{\mathcal{C}}^{a}\right),
$$

and it is a differentiable function on the phase space if (10) is satisfied and:

$$
\int_{\partial \Sigma} \mathrm{d}^{2} y \tilde{\varepsilon}^{a b} u_{a} \delta A_{b}=0 .
$$

This condition is satisfied for the allowed $\delta A_{a}$ on the boundary if the Lagrange multipliers $u_{a}$ vanish at the boundary, $\left.u_{a}\right|_{\partial \Sigma}=0$ or in the special case when $u_{a}=\left.\partial_{a} f\right|_{\partial \Sigma}$, where $f$ is an arbitrary function on the boundary. In this second case the condition (12) reduces to

$$
\int_{\partial \Sigma} \mathrm{d}^{2} y \tilde{\varepsilon}^{a b} \partial_{a} f \partial_{b} \lambda=-\int_{\partial \Sigma} \mathrm{d}^{2} y \tilde{\varepsilon}^{a b} f \nabla_{a} \nabla_{b} \lambda=0 .
$$

If either of these conditions are imposed, then the variation of the total Hamiltonian takes the form,

$$
\delta H_{\mathrm{T} 1}=\int_{\Sigma} \mathrm{d}^{3} x\left[-\left(\nabla_{a} \tilde{\Pi}^{a}\right) \delta \phi+u \delta \tilde{\Pi}_{\phi}-2 \tilde{\varepsilon}^{a b c}\left(\nabla_{b} u_{c}\right) \delta A_{a}+\left(u_{a}+\partial_{a} \phi\right) \delta \tilde{\Pi}^{a}\right] .
$$

Let us now consider the differentiability of the smeared constraints. The first one

$$
C_{1}[v]=\int_{\Sigma} \mathrm{d}^{3} x v \tilde{\Pi}_{\phi}
$$

is clearly differentiable, since it does not contain any derivative, while the second smeared constrained

$$
C_{2}\left[v_{a}\right]=\int_{\Sigma} \mathrm{d}^{3} x v_{a}\left(\tilde{\Pi}^{a}-\tilde{\varepsilon}^{a b c} F_{b c}\right),
$$

\footnotetext{
${ }^{1}$ From now on we shall use the symbol $\nabla_{a}$ for covariant derivatives, but it should be clear that all expression should be independent of the choice of derivative $\nabla_{a}$, since the theory does not depend on any background structure like a metric or connection.
} 
is differentiable only if a condition similar to (12) is imposed, namely

$$
\int_{\partial \Sigma} \mathrm{d}^{2} y \tilde{\varepsilon}^{a b} v_{a} \delta A_{b}=0
$$

that again leads to $\left.v_{a}\right|_{\partial \Sigma}=0$ or $v_{a}=\left.\partial_{a} g\right|_{\partial \Sigma}$. Allowed test functions are the ones that satisfy one of these restrictions. Then,

$$
\delta C_{2}=\int_{\Sigma} \mathrm{d}^{3} x\left[-2 \tilde{\varepsilon}^{a b c}\left(\nabla_{b} v_{c}\right) \delta A_{a}+v_{a} \delta \tilde{\Pi}^{a}\right]
$$

We can now analyze the consistency conditions of the constraints by computing

$$
\begin{aligned}
\left\{C_{1}[v], H_{\mathrm{T} 1}\right\} & \approx 0 \quad \Rightarrow \quad \tilde{\mathcal{C}}_{3}:=\nabla_{a} \tilde{\Pi}^{a} \approx 0, \\
\left\{C_{2}\left[v_{a}\right], H_{\mathrm{T} 1}\right\} & \approx 0 \quad \Rightarrow \quad \int_{\partial \Sigma} \mathrm{d}^{2} y \tilde{\varepsilon}^{a b} v_{a}\left(u_{b}+\partial_{b} \phi\right)=0 .
\end{aligned}
$$

The secondary constraint $\tilde{\mathcal{C}}_{3}$ is not independent, since $\tilde{\mathcal{C}}_{3}=\nabla_{a} \tilde{\mathcal{C}}^{a}$. The condition (20) is satisfied for all allowed $u_{a}$ and $v_{a}$. With this, we can conclude that there are no extra (independent) constraints for the system.

In order to further classify the constraints, we compute their Poisson brackets to obtain the algebra of constraints,

$$
\begin{aligned}
\left\{C_{1}[v], C_{1}[w]\right\} & =\left\{C_{1}[v], C_{2}\left[w_{a}\right]\right\}=0, \\
\left\{C_{2}\left[v_{a}\right], C_{2}\left[w_{b}\right]\right\} & =2 \int_{\partial \Sigma} \mathrm{d}^{2} y \tilde{\varepsilon}^{a b} v_{a} w_{b}=0 .
\end{aligned}
$$

Thus, the constraints are first class for all allowed test functions. Let us now perform a quick counting of (local) degrees of freedom. We started with 8 phase space degrees of freedom $\left(\phi, \tilde{\Pi}_{\phi} ; A_{a}, \tilde{\Pi}^{a}\right)$, and we have seen that there are four independent first class constraints $\left(\tilde{\mathcal{C}}, \tilde{\mathcal{C}}^{a}\right)$. Thus, there are $8-2 \cdot 4=0$ local degrees of freedom, which means that if there are any degrees of freedom, they would have to arise as would-be gauge degrees of freedom at the boundary. That will, of course, depend on both the boundary conditions and on the topology of the spacetime region. In our case we have no boundary degrees of freedom.

Let us now repeat the Dirac analysis for a second possible version of the canonical Hamiltonian.

\section{Second approach}

We can obtain this second way of representing the Hamiltonian if we substitute $\tilde{\Pi}^{a}=\tilde{\varepsilon}^{a b c} F_{b c}$ and $\tilde{\Pi}_{\phi}=0$ in $(6)$. The canonical Hamiltonian becomes

$$
H_{\mathrm{C} 2}=\int_{\Sigma} \mathrm{d}^{3} x \tilde{\varepsilon}^{a b c} F_{b c} \partial_{a} \phi=H_{\mathrm{C} 1}+C_{2}\left[\partial_{a} \phi\right] .
$$

Its variation is

$$
\delta H_{\mathrm{C} 2}=-\int_{\Sigma} \mathrm{d}^{3} x \tilde{\varepsilon}^{a b c}\left[2\left(\nabla_{b} \nabla_{a} \phi\right) \delta A_{c}+\nabla_{a} F_{b c} \delta \phi\right]+\int_{\partial \Sigma} \mathrm{d}^{2} y \tilde{\varepsilon}^{a b}\left(-2 \partial_{a} \phi \delta A_{b}+F_{a b} \delta \phi\right),
$$

so that the bulk term vanishes identically and the variation reduces to a boundary term.

The variation of $H_{\mathrm{T} 2}$ is well defined if the boundary term in $\delta H_{\mathrm{C} 2}$ vanishes, so we need to impose the condition (10), in this case

$$
\delta H_{\mathrm{T} 2}=\int_{\Sigma} \mathrm{d}^{3} x\left[u \delta \tilde{\Pi}_{\phi}+2 \tilde{\varepsilon}^{a b c}\left(\nabla_{b} u_{c}\right) \delta A_{a}+u_{a} \delta \tilde{\Pi}^{a}\right],
$$

for all allowed multipliers. Now, the consistency conditions and algebra of the constraints can be found straightforwardly,

$$
\begin{aligned}
\left\{C_{1}[v], H_{\mathrm{T} 2}\right\} & =0, \\
\left\{C_{2}\left[v_{a}\right], H_{\mathrm{T} 2}\right\} & =0, \\
\left\{C_{2}\left[v_{a}\right], C_{2}\left[u_{a}\right]\right\} & =0 .
\end{aligned}
$$

Note that in this case the consistency condition of $C_{1}$ is identically fulfilled, it does not produce any secondary constraint, as was the case in the first approach (even when that secondary constraint was not independent). 


\section{Third approach}

In this third approach, we perform an integration by parts in (23) and obtain a contribution only from the boundary

$$
H_{\mathrm{C} 3}=\int_{\partial \Sigma} \mathrm{d}^{2} y \tilde{\varepsilon}^{a b} F_{a b} \phi .
$$

Its variation is given by

$$
\delta H_{\mathrm{C} 3}=\int_{\partial \Sigma} \mathrm{d}^{2} y \tilde{\varepsilon}^{a b}\left(-2 \partial_{a} \phi \delta A_{b}+F_{a b} \delta \phi\right) .
$$

This is the same as in (24), so that $H_{\mathrm{T} 3}$ is well defined if the condition (10) is satisfied and for all allowed multipliers, just like in our second approach.

Note that the form of the canonical Hamiltonian is the same as in the Chern-Simons theory (see below), defined on $\partial \Sigma$, but since the total Hamiltonian of the Pontryagin theory is defined on $\Sigma$ the boundary terms in its variation should vanish. If we had started from the Chern-Simons theory on a three dimensional manifold $I \times \partial \Sigma$, we would have arrived at the canonical Hamiltonian (29), but the momenta and the constraints would have been different than in Pontryagin theory, and the corresponding total Hamiltonian would be defined on $\partial \Sigma$ (see below). This third approach makes it easier to compare to the case we shall consider in the next section, namely an Abelian Chern-Simons theory on $I \times \partial \Sigma$.

\section{B. Observables}

One of the main goals of this article is to compare two apparently distinct theories, namely Pontryagin on the bulk and Chern-Simons on the boundary. While we know that they are equivalent at the level of the action, we have two very different canonical descriptions, for the simple fact that they are defined on different spaces, one being the boundary of the other. One expects that, while the basic variables and the structure of the constraints might be different, one should still be able to describe the same "physics". It is natural then to consider physical observables, and the algebra they satisfy, as a way of matching both theories. Of course this strategy is not new and is pursued in the context of, for instance, dualities a la AdS/CFT [26 28].

General considerations on diffeomorphism invariant theories allow one to conclude that physical observables, when written as integrals over a hyper-surface $\Sigma$ of an integrand that depends (locally) on the fields and finite derivatives, have contributions only from the boundaries of $\Sigma$ (see for instance the discussion in [18]). Thus, it is natural to follow that strategy first proposed by Regge and Teitelboim [19], as we now describe. We shall try to construct observables as boundary terms that should be added to smeared first class constraints in order to make them differentiable, without imposing any conditions on the multipliers. In this case, since

$$
\delta C_{2}\left[u_{a}\right]=\int_{\Sigma} \mathrm{d}^{3} x\left[-2 \tilde{\varepsilon}^{a b c}\left(\nabla_{b} v_{c}\right) \delta A_{a}+v_{a} \delta \tilde{\Pi}^{a}\right]+\int_{\partial \Sigma} \mathrm{d}^{2} y \tilde{\varepsilon}^{a b} v_{a} \delta A_{b},
$$

we see that the functional

$$
Q\left[w_{a}\right]:=C_{2}\left[w_{a}\right]-\int_{\partial \Sigma} \mathrm{d}^{2} y \tilde{\varepsilon}^{a b} w_{a} A_{b},
$$

is differentiable for an arbitrary one-form $w_{a}$. In order to be an observable its Poisson brackets with the constraints should vanish. First note that $\left\{Q\left[w_{a}\right], C_{1}[v]\right\}=0$ and

$$
\left\{Q\left[w_{a}\right], C_{2}\left[u_{b}\right]\right\}=2 \int_{\partial \Sigma} \mathrm{d}^{2} y \tilde{\varepsilon}^{a b} w_{a} u_{b} .
$$

This Poisson bracket vanishes for: 1) arbitrary $w_{a}$ on $\partial \Sigma$ in the case when $u_{b}=\left.0\right|_{\partial \Sigma}$ or 2) for $w_{a}=\left.0\right|_{\partial \Sigma}$ or $w_{a}=\left.\partial_{a} f\right|_{\partial \Sigma}$ when $u_{a}=\left.\partial_{a} g\right|_{\partial \Sigma}$. As a result $Q\left[w_{a}\right]$ is an observable only when $w_{a}$ satisfy the same conditions as $u_{b}$ on $\partial \Sigma$. The first case, when $w_{a}=\left.0\right|_{\partial \Sigma}$, is trivial since $Q\left[w_{a}\right] \approx 0$. In the second case, when $w_{a}=\left.\partial_{a} f\right|_{\partial \Sigma}$, we have

$$
Q[f] \approx-\int_{\partial \Sigma} \mathrm{d}^{2} y \tilde{\varepsilon}^{a b} \partial_{a} f A_{b}=\frac{1}{2} \int_{\partial \Sigma} \mathrm{d}^{2} y f \tilde{\varepsilon}^{a b} F_{a b}=0,
$$

due to the condition (10). As a result, this construction leads to trivial observables. 
Another possible strategy for finding non trivial observables is to consider a family of linear functionals as

$$
\pi\left[g_{a}\right]=\int_{\Sigma} \mathrm{d}^{3} x g_{a} \tilde{\Pi}^{a},
$$

where $g_{a}$ is an arbitrary one-form on $\Sigma$. In this case we have

$$
\left\{\pi\left[g_{a}\right], C_{2}\left[v_{c}\right]\right\}=-2 \int_{\Sigma} \mathrm{d}^{3} x \tilde{\varepsilon}^{a b c}\left(\nabla_{b} v_{c}\right) g_{a},
$$

for all allowed $v_{c}$. This can be rewritten as

$$
\left\{\pi\left[g_{a}\right], C_{2}\left[v_{c}\right]\right\}=2 \int_{\Sigma} \mathrm{d}^{3} x \tilde{\varepsilon}^{a b c}\left(\nabla_{b} g_{a}\right) v_{c}-2 \int_{\partial \Sigma} \mathrm{d}^{2} y \tilde{\varepsilon}^{a b} v_{a} g_{b},
$$

and this expression does not vanish unless $g_{a}=\nabla_{a} f$, that leads to the family of linear observables of the form

$$
\mathcal{O}[f]=\int_{\Sigma} \mathrm{d}^{3} x\left(\nabla_{a} f\right) \tilde{\Pi}^{a} \approx \int_{\partial \Sigma} \mathrm{d} S_{a} f \tilde{\Pi}^{a}=0
$$

due to (10), again resulting in trivial observables.

The other family of linear functionals could be constructed as

$$
q\left[h^{a}\right]=\int_{\Sigma} \mathrm{d}^{3} x h^{a} A_{a},
$$

but in this case

$$
\left\{q\left[h^{a}\right], C_{2}\left[v_{b}\right]\right\}=\int_{\Sigma} \mathrm{d}^{3} x h^{a} v_{a},
$$

so $q\left[h^{a}\right]$ is not an observable for any election of $h^{a}$.

Let us also consider a non-linear functional of the form

$$
N[f]=\int_{\Sigma} \mathrm{d}^{3} x f\left(\nabla_{a} \phi\right) \tilde{\Pi}^{a} .
$$

This expression is differentiable

$$
\delta N=\int_{\Sigma} \mathrm{d}^{3} x\left[f\left(\nabla_{a} \phi\right) \delta \tilde{\Pi}^{a}-\nabla_{a}\left(f \tilde{\Pi}^{a}\right) \delta \phi\right]+\int_{\partial \Sigma} \mathrm{d} S_{a} f \tilde{\Pi}^{a} \delta \phi,
$$

since the boundary term vanishes due to (10). Now,

$$
\left\{N[f], C_{1}[g]\right\} \approx-\int_{\Sigma} \mathrm{d}^{3} x g \tilde{\Pi}^{a} \nabla_{a} f,
$$

and it vanishes only if $f=$ const. In this case,

$$
\left\{N[f], C_{2}\left[u_{b}\right]\right\} \approx 2 f \int_{\partial \Sigma} \mathrm{d}^{2} y \tilde{\varepsilon}^{a b}\left(\nabla_{a} \phi\right) u_{b},
$$

and it vanishes for all allowed $u_{b}$. For any constant $f$, we again have a trivial observable, since $N[f]=f H_{\mathrm{C} 1}$, and

$$
N[f]=f \int_{\Sigma} \mathrm{d}^{3} x\left(\nabla_{a} \phi\right) \tilde{\Pi}^{a} \approx f \int_{\partial \Sigma} \mathrm{d} S_{a} \tilde{\Pi}^{a} \phi=0 .
$$

Note that the fact $H_{\mathrm{C} 1} \approx 0$ indicates that the theory is invariant under diffeomorphisms.

Let us examine yet another non-linear functional

$$
P\left[g^{a}\right]=\int_{\Sigma} \mathrm{d}^{3} x g^{a} \tilde{\Pi}^{b} F_{a b} .
$$


This functional is differentiable for $r_{b} g^{b}=\left.0\right|_{\partial \Sigma}$. Then,

$$
\left\{P\left[g^{a}\right], C_{2}\left[u_{b}\right]\right\} \approx \int_{\Sigma} \mathrm{d}^{3} x g^{d} \tilde{\varepsilon}^{a b c}\left[2 F_{a d} \nabla_{b} u_{c}-F_{b c}\left(\nabla_{a} u_{d}-\nabla_{d} u_{a}\right)\right],
$$

and it does not vanish for any $g^{a} \neq 0$, so it is not a physical observable.

We started with a theory that is known not to have any local degrees of freedom, so the only possibility, when there is a boundary present, is that new would-be gauge degrees of freedom arise on the boundary. In some cases, these have been referred to as "edge states". By considering a time-like boundary that has the topology $I \times S^{2}$, one expects a theory of Abelian flat connections on the boundary to have no degrees of freedom. That is indeed corroborated by the fact that a small sample of simple observables are trivial. While we have not provided an exhaustive list of candidates, one should expect that (the gradients of) linear and the simplest non-linear observables should span, locally, the co-tangent space of the phase space. Thus, this suggests that there are no non-trivial observables, and therefore, no local physical degrees of freedom at the boundary. As mentioned before, if the topology of the boundary were non-trivial, in the sense that $H^{1}(\partial \Sigma) \neq 0$, we would have non-trivial global degrees of freedom that could be explored, for instance, by Wilson-loops around homotopically non-trivial curves.

\section{Generator of gauge transformations}

Let us now find the generators of gauge transformations, which are constructed as a linear combination of first class constraints

$$
G\left[\epsilon_{1}, \epsilon_{2}, \eta_{a}\right]=\int_{\Sigma} \mathrm{d}^{3} x\left[\epsilon_{1} \tilde{\Pi}_{\phi}+\epsilon_{2} \nabla_{a} \tilde{\Pi}^{a}+\eta_{a}\left(\tilde{\Pi}^{a}-\tilde{\varepsilon}^{a b c} F_{b c}\right)\right]
$$

Though the constraint $\nabla_{a} \tilde{\Pi}^{a} \approx 0$ is not an independent one, we include it as a part of the generator, and we shall show that this proposal generates the usual gauge and diffeomorphism symmetries of the theory. The generator is differentiable if the condition (10) is satisfied and $\left.\eta_{a}\right|_{\partial \Sigma}=0$ or $\eta_{a}=\left.\partial_{a} g\right|_{\partial \Sigma}$. The corresponding gauge transformations are

$$
\begin{aligned}
\delta \phi & =\epsilon_{1}, \quad \delta \tilde{\Pi}_{\phi}=0, \\
\delta A_{a} & =-\nabla_{a} \epsilon_{2}+\eta_{a}, \\
\delta \tilde{\Pi}^{a} & =2 \tilde{\varepsilon}^{a b c} \nabla_{b} \eta_{c} .
\end{aligned}
$$

Depending on the particular choices of the smearing functions, that can be phase space independent or dependent (live), one has different classes of gauge transformations.

We shall consider three cases:

1. $U(1)$ gauge symmetry is obtained for the choice $\epsilon_{1}=£_{t} \epsilon, \epsilon_{2}=-\epsilon$ and $\eta_{a}=0$, then

$$
\begin{aligned}
\delta_{\epsilon} \phi & =£_{t} \epsilon, \\
\delta_{\epsilon} A_{a} & =\nabla_{a} \epsilon .
\end{aligned}
$$

Here we have used the result by [29] that states that, in order to obtain (spacetime) gauge transformations, one has to choose the parameter $\epsilon_{1}$ to be the time derivative of the parameter involved in spatial transformations.

2. Spatial diffeomorphisms are obtained for the phase space dependent choice $\epsilon_{1}=£_{\xi} \phi, \epsilon_{2}=-\xi^{a} A_{a}$ and $\eta_{a}=\xi^{b} F_{b a}$ (note that $\left.\eta_{a}\right|_{\partial \Sigma}=0$ ), where $\xi^{a} t_{a}=0$. Then, we have

$$
\begin{aligned}
\delta_{\xi} \phi & =£_{\xi} \phi, \\
\delta_{\xi} A_{a} & =\partial_{a}\left(\xi^{b} A_{b}\right)+\xi^{b} F_{b a}=\left(\nabla_{a} \xi^{b}\right) A_{b}+\xi^{b} \nabla_{b} A_{a}=£_{\xi} A_{a} .
\end{aligned}
$$

On the other hand

$$
\begin{aligned}
\delta_{\xi} \tilde{\Pi}^{a} & =2 \tilde{\varepsilon}^{a b c} \nabla_{b}\left(\xi^{d} F_{d c}\right) \approx \tilde{\varepsilon}^{a b c} \xi_{d c n} \nabla_{b}\left(\xi^{d} \tilde{\Pi}^{n}\right) \\
& =\nabla_{b}\left(\xi^{b} \tilde{\Pi}^{a}-\xi^{a} \tilde{\Pi}^{b}\right)=\nabla_{b}\left(\xi^{b} \tilde{\Pi}^{a}\right)-\left(\nabla_{b} \xi^{a}\right) \tilde{\Pi}^{b}-\xi^{a} \nabla_{b} \tilde{\Pi}^{b} \approx £_{\xi} \tilde{\Pi}^{a},
\end{aligned}
$$

since $\xi_{d c n} \tilde{\Pi}^{n} \approx 2 F_{d c}$. Thus, $A_{a}$ transforms like a one form and $\tilde{\Pi}^{a}$ like a vector density of weight 1 under spatial diffeomorphisms. 
3. 'Time-like' diffeomorphisms, or 'time evolution' are obtained for $\epsilon_{1}=£_{t} \phi, \epsilon_{2}=-\phi$ and $\eta_{a}=£_{t} A_{a}-\nabla_{a} \phi$. Then, we have

$$
\begin{array}{r}
\delta_{t} \phi=£_{t} \phi, \\
\delta_{t} A_{a}=£_{t} A_{a} .
\end{array}
$$

It is straightforward to check that the transformations for the canonical momenta have similar expressions.

In the next section we shall consider the theory as a boundary theory defined on $I \times \partial \Sigma$.

\section{HAMILTONIAN ANALYSIS OF THE CHERN-SIMONS THEORY ON THE BOUNDARY}

Integration by parts in the Pontryagin action (11), leads to the action of the Chern-Simons theory on the boundary $\mathcal{B}=I \times \partial \Sigma$ given by

$$
S_{\mathrm{CS}}=-\frac{1}{2} \int_{\mathcal{B}} \mathrm{d}^{3} x \tilde{\varepsilon}^{a b c} A_{a} F_{b c} .
$$

Even when the boundary of the spacetime region where the Pontryagin theory is defined includes the initial and final "spatial" hypersurfaces, it is customary to neglect the contributions to the action from those hypersurfaces since in the action principle the variations of the fields are always vanishing. We shall take this viewpoint here. This section has three parts. In the first one, we perform the canonical decomposition of the Chern-Simon theory on the "time-like" boundary. ${ }^{2}$ In the second one we consider physical observables and in the third part we construct the generators of gauge transformations.

\section{A. Canonical decomposition}

Since $\tilde{\varepsilon}^{a b c}=3 t^{[a} \tilde{\varepsilon}^{b c]}$ we have

$$
S_{\mathrm{CS}}=-\frac{1}{2} \int_{\mathcal{B}} \mathrm{d}^{3} x\left(\tilde{\varepsilon}^{b c} \phi F_{b c}+2 \tilde{\varepsilon}^{a b} t^{c} F_{b c} A_{a}\right)=-\int_{\mathcal{B}} \mathrm{d}^{3} x \tilde{\varepsilon}^{a b}\left[\left(£_{t} A_{a}\right) A_{b}+F_{a b} \phi\right],
$$

where $\phi=t^{a} A_{a}$ and $t^{c} F_{b c}=-£_{t} A_{b}+\nabla_{b} \phi$. The corresponding momenta are

$$
\tilde{\Pi}^{a}=-\tilde{\varepsilon}^{a b} A_{b},
$$

and the canonical Hamiltonian is

$$
H_{\mathrm{CS}}=\int_{\partial \Sigma} \mathrm{d}^{2} y\left[\left(£_{t} \phi\right) \tilde{\Pi}_{\phi}+\left(£_{t} A_{a}\right) \tilde{\Pi}^{a}-\mathcal{L}_{C S}\right] \approx \int_{\partial \Sigma} \mathrm{d}^{2} y \tilde{\varepsilon}^{a b} F_{a b} \phi .
$$

Note that $H_{\mathrm{CS}}$ has the same form as the canonical Hamiltonian of the third approach on the bulk (29), but now it is differentiable for arbitrary variations $\delta A_{a}$ and $\delta \phi$, since there are no boundary terms (the boundary of the boundary is zero).

The theory has three primary constraints

$$
\begin{aligned}
\tilde{\Pi}_{\phi} & :=t_{a} \tilde{\Pi}^{a} \approx 0, \\
\varphi^{a} & :=\tilde{\Pi}^{a}+\tilde{\varepsilon}^{a b} A_{b} \approx 0 .
\end{aligned}
$$

The constraints $\varphi^{a}$ are second class, since

$$
\left\{\varphi^{a}(y), \varphi^{b}\left(y^{\prime}\right)\right\}=2 \tilde{\varepsilon}^{a b} \delta^{2}\left(y, y^{\prime}\right) .
$$

\footnotetext{
2 Note that we are writing "space-like" and "time-like" in analogy with theories where a metric exists, even when in this case there is no metric and therefore no notion of causality.
} 
The total Hamiltonian is

$$
H_{\mathrm{TCS}}=\int_{\partial \Sigma} \mathrm{d}^{2} y\left[\tilde{\varepsilon}^{a b} F_{a b} \phi+\lambda \tilde{\Pi}_{\phi}+\lambda_{a}\left(\tilde{\Pi}^{a}+\tilde{\varepsilon}^{a b} A_{b}\right)\right],
$$

and is differentiable for arbitrary fields variations and for arbitrary multipliers $\lambda$ and $\lambda_{a}$. The consistency conditions for primary constraints lead to

$$
\begin{aligned}
\left\{\tilde{\Pi}_{\phi}, H_{\mathrm{TCS}}\right\} & =-\tilde{\varepsilon}^{a b} F_{a b} \approx 0, \\
\left\{\varphi^{a}, H_{\mathrm{TCS}}\right\} & =2 \tilde{\varepsilon}^{a b}\left(\partial_{b} \phi-\lambda_{b}\right) .
\end{aligned}
$$

As a result, there is a secondary constraint

$$
\Psi:=\tilde{\varepsilon}^{a b} F_{a b} \approx 0
$$

and the multipliers $\lambda_{a}$ are determined as $\lambda_{a}=\partial_{a} \phi$. Now, the consistency condition for $\Psi$ is trivially fulfilled. If we consider the smeared constraint

$$
\Psi[v]=\int_{\partial \Sigma} \mathrm{d}^{2} y v \tilde{\varepsilon}^{a b} F_{a b} \approx 0
$$

its variation is given by

$$
\delta \Psi[v]=-2 \int_{\partial \Sigma} \mathrm{d}^{2} y \tilde{\varepsilon}^{a b} \partial_{a} v \delta A_{b}
$$

Then,

$$
\left\{\Psi[v], H_{\mathrm{TCS}}\right\}=2 \int_{\partial \Sigma} \mathrm{d}^{2} y \tilde{\varepsilon}^{a b} \partial_{a} \lambda_{b}=2 \int_{\partial \Sigma} \mathrm{d}^{2} y \tilde{\varepsilon}^{a b} \partial_{a} \partial_{b} \phi=0,
$$

and there are no tertiary constraints.

Let us now analyze the constrained structure of the theory. There are two first class constraints

$$
\begin{aligned}
\tilde{\Pi}_{\phi} & \approx 0, \\
\mathcal{C} & :=\nabla_{a} \varphi^{a}-\Psi=\nabla_{a}\left(\tilde{\Pi}^{a}-\tilde{\varepsilon}^{a b} A_{b}\right) \approx 0,
\end{aligned}
$$

and two second class constraints

$$
\varphi^{a} \approx 0
$$

The counting of local degrees of freedom leads to $3 \cdot 2-2 \cdot 2-2=0$.

Note that, after introducing the expression for $\lambda_{a}=\partial_{a} \phi$ and performing the integration by parts, the total Hamiltonian (63) can be rewritten as a combination of the first class constraints

$$
H_{\mathrm{TCS}}=\int_{\partial \Sigma} \mathrm{d}^{2} y\left(-\phi \mathcal{C}+\lambda \tilde{\Pi}_{\phi}\right)
$$

We can obtain the equations of motion from the canonical action

$$
S_{\mathrm{CCS}}=\int_{\mathcal{B}} \mathrm{d}^{3} x\left[\left(£_{t} \phi\right) \tilde{\Pi}_{\phi}+\left(£_{t} A_{a}\right) \tilde{\Pi}^{a}+\phi \mathcal{C}-\lambda \tilde{\Pi}_{\phi}\right]
$$

Then

$$
\begin{aligned}
& \frac{\delta S_{\mathrm{C}}}{\delta \tilde{\Pi}_{\phi}}=0 \quad \Rightarrow \quad £_{t} \phi=\lambda, \\
& \frac{\delta S_{\mathrm{C}}}{\delta \tilde{\Pi}^{a}}=0 \quad \Rightarrow \quad £_{t} A_{a}=\nabla_{a} \phi .
\end{aligned}
$$

The last equation implies that $t^{a} F_{a b}=£_{t} A_{b}-\nabla_{b} \phi=0$. Thus, we recover the Lagrangian equations of motion that state that the $3 D$ connection $A_{a}$ on $\mathcal{B}$ is flat. The pullback of $F_{a b}$ to $\partial \Sigma$ is zero due to the constraint (66), and the 
transverse part vanishes as well as shown by the previous equation. Thus, the full $3 D$ curvature vanishes $F_{a b}=0$, recovering the covariant equations of motion for the Chern-Simons theory.

Let us end this part with a remark. We have performed a complete Dirac analysis of the Abelian Chern-Simons theory on $\mathcal{B}$, sometimes referred as a 'true-Dirac' analysis. This has to be compared to a reduced analysis that can sometimes be found in the literature, for instance in [6] and references therein. In this latter analysis, one solves the second class constraint (61) for $\tilde{\Pi}^{a}$, and gauge fixes the first class constraint (60) in order to eliminate $\phi$ and its conjugate momentum as dynamical variables. The end result is that the (partially) reduced phase space consists of $U(1)$ connections $A_{a}$, subject to the Dirac-Poisson bracket $\left\{A_{a}(x), A_{b}(y)\right\}_{\mathrm{D}}={\underset{\sim}{a b}}_{a b} \delta^{2}(x, y)$, and to the only remaining, first class constraint, $\Psi=\tilde{\varepsilon}^{a b} F_{a b} \approx 0$. The counting of local degrees of freedom, $2 \cdot 1-2 \cdot 1=0$ still yields zero. Needless to say, the final description of the physical theory is equivalent in both cases. We have chosen to perform the complete analysis in order to make contact with the variables and degrees of freedom of the bulk theory.

\section{B. Observables}

Just as we did in the previous section with the bulk theory, we shall attempt to construct observables for the boundary theory. We know that there are no local degrees of freedom, so if there are non-trivial degrees of freedom they have to come from topological considerations. Since we are choosing a rather trivial topology for the space $\mathcal{B}$, we do not expect to obtain non-trivial observables. Still it is a useful exercise to go through the process.

We start by constructing a linear functional

$$
\mathcal{O}\left[f_{a}\right]=\int_{\partial \Sigma} \mathrm{d}^{2} y f_{a} \tilde{\Pi}^{a}
$$

Its Poisson bracket with the smeared first class constraint $\mathcal{C}$

$$
C[g]=\int_{\partial \Sigma} \mathrm{d}^{2} y g \nabla_{a}\left(\tilde{\Pi}^{a}-\theta \tilde{\varepsilon}^{a b} A_{b}\right),
$$

is

$$
\left\{\mathcal{O}\left[f_{a}\right], C[g]\right\}=-\theta \int_{\partial \Sigma} \mathrm{d}^{2} y \tilde{\varepsilon}^{a b} f_{a} \nabla_{b} g
$$

and, since $g$ is an arbitrary function, $\mathcal{O}\left[f_{a}\right]$ is an observable for $f_{a}=\nabla_{a} f$, but it is a trivial one since $\nabla_{a} \tilde{\Pi}^{a}$ is a linear combination of constraints,

$$
\mathcal{O}[f]=\int_{\partial \Sigma} \mathrm{d}^{2} y\left(\nabla_{a} f\right) \tilde{\Pi}^{a}=-\int_{\partial \Sigma} \mathrm{d}^{2} y f \nabla_{a} \tilde{\Pi}^{a}=-\frac{1}{2} \int_{\partial \Sigma} \mathrm{d}^{2} y f\left(\mathcal{C}+\nabla_{a} \varphi^{a}\right) \approx 0 .
$$

In our search for observables, one can also consider other combinations. Let us first note that $A_{a} \tilde{\Pi}^{a} \approx-\theta \tilde{\varepsilon}^{a b} A_{a} A_{b}=$ 0 and $F_{a b} \approx 0$. Let us then construct another functional that also involves $\phi$ as follows,

$$
\mathcal{P}[f]=\int_{\partial \Sigma} \mathrm{d}^{2} y f\left(\nabla_{a} \phi\right) \tilde{\Pi}^{a} .
$$

In order to be an observable its Poisson bracket with $C_{1}[g]=\int_{\partial \Sigma} \mathrm{d}^{2} y g \tilde{\Pi}_{\phi}$ must vanish

$$
\left\{\mathcal{P}[f], C_{1}[g]\right\}=-\int_{\partial \Sigma} \mathrm{d}^{2} y g \nabla_{a}\left(f \tilde{\Pi}^{a}\right) \approx-\int_{\partial \Sigma} \mathrm{d}^{2} y g\left(\nabla_{a} f\right) \tilde{\Pi}^{a},
$$

and it vanishes only if $f=$ const, but in that case

$$
\mathcal{P}[f]=-f \int_{\partial \Sigma} \mathrm{d}^{2} y \phi\left(\nabla_{a} \tilde{\Pi}^{a}\right) \approx 0 .
$$

That is, we have found a trivial observable again. Thus, our limited search for physical observables takes us again to trivial observables. Note that since $\partial \Sigma$ does not have a boundary, we can not construct observables from the constraints by adding boundary terms. 


\section{Generator of gauge transformations}

To end this section, let us find the generators of gauge transformation in the boundary theory. The generators of gauge transformation are of the form

$$
G\left[\epsilon_{1}, \epsilon_{2}\right]=\int_{\partial \Sigma} \mathrm{d}^{2} y\left[\epsilon_{1} \tilde{\Pi}_{\phi}+\epsilon_{2} \nabla_{a}\left(\tilde{\Pi}^{a}-\tilde{\varepsilon}^{a b} A_{b}\right)\right],
$$

where $\epsilon_{1}$ and $\epsilon_{2}$ are arbitrary (possibly phase space dependent) functions. The corresponding gauge transformations are

$$
\begin{aligned}
\delta \phi & =\epsilon_{1}, \\
\delta A_{a} & =-\nabla_{a} \epsilon_{2} .
\end{aligned}
$$

We can consider three special cases:

1. $U(1)$ gauge transformations can be obtained, for the choice $\epsilon_{1}=£_{t} \epsilon$ and $\epsilon_{2}=-\epsilon$, then

$$
\begin{aligned}
\delta_{\epsilon} \phi & =£_{t} \epsilon, \\
\delta_{\epsilon} A_{a} & =\nabla_{a} \epsilon .
\end{aligned}
$$

2. Spatial diffeomorphisms. With the choice of field dependent parameter $\epsilon_{1}=£_{\xi} \phi$ and $\epsilon_{2}=-\xi^{a} A_{a}$, such that $\xi^{a} t_{a}=0$, we obtain

$$
\begin{aligned}
\delta_{\xi} \phi & =£_{\xi} \phi \\
\delta_{\xi} A_{a} & =£_{\xi} A_{a}+\xi^{b} F_{b a} \approx £_{\xi} A_{a},
\end{aligned}
$$

where the last equation is valid on the constrained phase space surface.

3. 'Time-like' diffeomorphisms. Time-like or time evolution diffeomorphisms can be obtained for $\epsilon_{1}=£_{t} \phi$ and $\epsilon_{2}=-\phi$. Thus,

$$
\begin{aligned}
\delta_{t} \phi & =£_{t} \phi, \\
\delta_{t} A_{a} & =£_{t} A_{a}+t^{b} F_{b a} \approx £_{t} A_{a},
\end{aligned}
$$

where the last equation is valid on-shell, that is, on the space of the solutions of the equations of motion.

Thus, we see that the physical theory is both "gauge invariant" and diffeomorphism invariant on $\mathcal{B}$, with no local degrees of freedom.

\section{DISCUSSION ON THE CANONICAL ANALYSIS: PONTRYAGIN VS. CHERN-SIMONS}

In this section we would like to discuss and comment on the similarities and differences one encounters in analyzing both the bulk and the boundary theories. We summarize these results in the Table I below.

The Pontryagin term is defined on a 4-dim. manifold $\mathcal{M}$ with boundary $\partial \mathcal{M}=\mathcal{B} \cup \Sigma_{1} \cup \Sigma_{2}$, where $\mathcal{B}=I \times \partial \Sigma$ is a "time-like" boundary, while the Chern-Simons term is defined on a 3 -dimensional $\mathcal{B}$. In the canonical Hamiltonian formulation of these theories the corresponding kinematical phase spaces are 8 and 6 dimensional, respectively. In the Pontryagin theory there are four constraints, all of them are first class, while in the Chern-Simons theory there are two first class constraints and two second class ones.

We found that the Hamiltonian of the Pontryagin theory is differentiable for arbitrary variations $\delta \phi$ only if $F_{a b}=0$ on the boundary $\partial \Sigma$. That condition appears also in the Chern-Simons theory, but in the form of a secondary constraint. Although we didn't a priori specify any boundary conditions, nor a condition on the variations that are allowed, the requirement of having a well defined Hamiltonian imposes a restriction on the variation of $A_{a}$; the only allowed variations are pure gauge.

When analysing the gauge symmetries that both theories posses, we see that they both have the same gauge symmetries, whose generators can be constructed out of the corresponding first class constraints. Again, even when the structure and the explicit form of the constraints is different in each case, we do recover in both cases, local $U(1)$ gauge transformations, spatial diffeomorphisms, and transverse ("time-like") diffeomorphisms. Thus, in both cases we recover a theory of gauge invariant, flat, and diffeomorphism invariant connections on $\partial \Sigma$. 


\begin{tabular}{|c|c|c|}
\hline & Pontryagin & Chern-Simons \\
\hline Action & $S_{P}=\frac{1}{4} \int_{\mathcal{M}} \mathrm{d}^{4} x \tilde{\varepsilon}^{a b c d} F_{a b} F_{c d}$ & $S_{C S}=-\frac{1}{2} \int_{\mathcal{B}} \mathrm{d}^{3} x \tilde{\varepsilon}^{a b c} A_{a} F_{b c}$ \\
\hline Canonical variables & 8 -dim. $\quad\left(\phi, \tilde{\Pi}_{\phi} ; A_{a}, \tilde{\Pi}^{a}\right)$ & 6-dim. $\quad\left(\phi, \tilde{\Pi}_{\phi} ; A_{a}, \tilde{\Pi}^{a}\right)$ \\
\hline $\begin{array}{l}\text { Constraints } \\
\text { Primary } \\
\text { Secondary }\end{array}$ & $\begin{array}{c}\tilde{\mathcal{C}}_{1}:=\tilde{\Pi}_{\phi}, \quad \tilde{\mathcal{C}}_{2}^{a}:=\tilde{\Pi}^{a}-\tilde{\varepsilon}^{a b c} F_{b c} \\
\tilde{\mathcal{C}}_{3}:=\nabla_{a} \tilde{\Pi}^{a}\end{array}$ & $\begin{array}{c}\tilde{\mathcal{C}}_{1}:=\tilde{\Pi}_{\phi}, \quad \varphi^{a}:=\tilde{\Pi}^{a}+\tilde{\varepsilon}^{a b} A_{b} \\
\Psi:=\tilde{\varepsilon}^{a b} F_{a b}\end{array}$ \\
\hline $\begin{array}{l}\text { 1st class } \\
\text { 2nd class }\end{array}$ & $\underset{\tilde{\mathcal{C}}_{1},}{\tilde{\mathcal{C}}_{2}^{a},} \underset{\text { none }}{\tilde{\mathcal{C}}_{3}}=\nabla_{a} \tilde{\mathcal{C}}_{2}^{a}$ & $\begin{array}{c}\tilde{\mathcal{C}}_{1}, \mathcal{C}:=\nabla_{a} \varphi^{a}-\Psi \\
\varphi^{a}\end{array}$ \\
\hline $\begin{array}{l}\text { Total Hamiltonian } \\
\text { Diff. conditions }\end{array}$ & $\begin{array}{c}H_{\mathrm{T} 1}=\int_{\Sigma} \mathrm{d}^{3} x\left(u \tilde{\mathcal{C}}_{1}-\phi \tilde{\mathcal{C}}_{3}+u_{a} \tilde{\mathcal{C}}_{2}^{a}\right) \\
\text { On } \partial \Sigma: F_{a b}=0 ; u_{a}=0 \text { or } u_{a}=\partial_{a} f\end{array}$ & $H_{T C S}=\underset{\text { none }}{\int_{\partial \Sigma} \mathrm{d}^{2} y\left(u \tilde{\mathcal{C}}_{1}-\phi \mathcal{C}\right)}$ \\
\hline $\begin{array}{l}\text { Gauge generator } \\
\text { Diff. conditions }\end{array}$ & $\begin{array}{c}G=\int_{\Sigma} \mathrm{d}^{3} x\left[\epsilon_{1} \tilde{\mathcal{C}}_{1}+\epsilon_{2} \tilde{\mathcal{C}}_{3}+\eta_{a} \tilde{\mathcal{C}}_{2}^{a}\right] \\
\text { On } \partial \Sigma: F_{a b}=0 ; \eta_{a}=0 \text { or } \eta_{a}=\partial_{a} f\end{array}$ & $G=\underset{\text { none }}{\int_{\partial \Sigma} \mathrm{d}^{2} y\left[\epsilon_{1} \tilde{\mathcal{C}}_{1}+\epsilon_{2} \mathcal{C}\right]}$ \\
\hline $\begin{array}{c}\text { Gauge symmetries } \\
\text { U(1) } \\
\text { Spatial Diffeo. } \\
\text { Time-like Diffeo. }\end{array}$ & $\begin{array}{c}\epsilon_{1}=£_{t} \epsilon, \epsilon_{2}=-\epsilon, \eta_{a}=0 \\
\epsilon_{1}=£_{\xi} \phi, \epsilon_{2}=-\xi^{a} A_{a}, \eta_{a}=\xi^{b} F_{b a} \\
\epsilon_{1}=£_{t} \phi, \epsilon_{2}=-\phi, \eta_{a}=£_{t} A_{a}-\nabla_{a} \phi\end{array}$ & $\begin{array}{c}\epsilon_{1}=£_{t} \epsilon, \epsilon_{2}=-\epsilon \\
\epsilon_{1}=£_{\xi} \phi, \epsilon_{2}=-\xi^{a} A_{a} \\
\epsilon_{1}=£_{t} \phi, \epsilon_{2}=-\phi\end{array}$ \\
\hline Observables & $\begin{array}{c}\mathcal{O}_{1}[f]:=\int_{\Sigma} \mathrm{d}^{3} x\left(\nabla_{a} f\right) \tilde{\Pi}^{a} \approx 0 \\
\mathcal{O}_{2}:=\int_{\Sigma} \mathrm{d}^{3} x\left(\nabla_{a} \phi\right) \tilde{\Pi}^{a} \approx 0\end{array}$ & $\begin{array}{c}\tilde{\mathcal{O}}_{1}[f]:=\int_{\partial \Sigma} \mathrm{d}^{2} y\left(\nabla_{a} f\right) \tilde{\Pi}^{a} \approx 0 \\
\tilde{\mathcal{O}}_{2}:=\int_{\partial \Sigma} \mathrm{d}^{2} y\left(\nabla_{a} \phi\right) \tilde{\Pi}^{a} \approx 0\end{array}$ \\
\hline
\end{tabular}

TABLE I: Comparison between canonical descriptions of Pontryagin and Chern-Simons theories

Finally, we constructed and analysed physical observables for both theories, and found in both cases the same type of observables, even though all of them are trivial, due to the triviality of the topologies chosen. Here we have taken $\Sigma$ such that $\partial \Sigma=S^{2}$. Had we chosen a non-trivial boundary, we would have non-trivial global degrees of freedom (accessible through Wilson-loops around homotopically nontrivial loops, for instance). As we discussed in the previous section, the observables in both theories can be written as integrals over the boundary, in such a way that can easily be related and identified with each other (note that in the table the Pontryagin observables have been rewritten as bulk integrals).

Let us end this with a remark. As we mentioned in the introduction, in the manuscript 25] these two theories have been studied in the canonical formalism (for $S O(3,1)$ ), which makes their results very close to ours. However, there are important and subtle differences that we enumerate as follows: (1) Here the Pontryagin theory is actually defined on a manifold with a boundary and the Chern-Simons theory is defined on this boundary; (2) We rewrite the Hamiltonian of the Pontryagin theory in three equivalent ways, inspect the resulting boundary conditions and relate them to constraints of the Chern- Simons theory; (3) We use a fully covariant approach in the canonical decomposition, without fixing a foliation nor a coordinate system and; (4) We construct observables in both theories that allow us to make direct comparison between the two theories. 


\section{OUTLOOK}

Gauge theories defined on regions with boundaries are without doubt, of wide physical interest. A complete canonical description of those theories based on the Dirac algorithm is, in our opinion, still lacking (see however [8, 30]). This is particularly noticeable when one has a theory defined by an action with both bulk and boundary contributions, and this later possesses time derivatives of the fundamental variables. In this case, the corresponding canonical momenta have contributions from both the bulk and the boundary, and one has then to properly define the symplectic and Poisson structures of the theory. Our ultimate goal is to provide a systematic analysis of such scenarios.

Here we have considered a simpler case, where the theory can be alternatively seen as a bulk theory or as one defined on the boundary. What we have here learned will be useful in the next step that is coupling the Pontryagin bulk theory to a Maxwell field (or in the non-Abelian case, Yang Mills), and thus obtaining the well studied case of Yang-Mills with a $\theta$ term. Here the challenge is to alternatively describe the resulting theory as a pure bulk theory or as having a bulk and a boundary contribution. Of course, both descriptions should coincide and one should then have an appropriate "dictionary" (to borrow a term from AdS/CFT) to compare both theories. The relation between the two descriptions in our simple example should then be helpful to construct such dictionary for the Maxwell-Pontryagin case.

Of course, the system we have here analysed is rather simple, and in certain sense, trivial. Still, this feature that allows one to "solve it", is relevant since one can then precisely point out how to relate and compare the two theories defined on different manifolds, bulk and boundary, and show their equivalence. We have also pointed out certain subtleties that have allowed us to make this comparison, such as a careful analysis of the boundary conditions imposed in order to make the canonical description of the bulk theory well defined, and how those boundary conditions are related to (secondary) constraints on the boundary. While such relations had been suggested before in other systems, we feel that the simplicity of this one allows for a very clear understanding of this correspondence.

We hope that these results will be useful in our program of understanding, from the "Dirac" perspective, gauge systems defined on regions with boundaries. This viewpoint has to be contrasted to, say, the geometrical approach of [24], that can be seen as complementary to ours. We shall report some of those results elsewhere [31].

\section{Acknowledgments}

We would like to thank I. Rubalcava-Garcia for discussions. This work was in part supported by CONACyT 0177840 and PAPIIT IN100218 grants and by CIC, UMSNH.

[1] A.P. Balachandran, L. Chandar and E. Ercolessi, "Edge states in gauge theories: Theory, interpretations and predictions", Int. J. Mod. Phys. A10, 1969, 1995. arXiv:hep-th/9411164 [hep-th]]

[2] E. Witten, "Quantum field theory and the Jones polynomial", Commun. Math. Phys. 121, 351, 1989.

[3] S. Elitzur, G.W. Moore, A. Schwimmer and N. Seiberg, "Remarks on the canonical quantization of the Chern-SimonsWitten theory", Nucl.Phys. B326, 108, 1989.

[4] G.W. Moore and N. Seiberg, "Taming the conformal zoo", Phys.Lett. B220, 422, 1989.

[5] M. Bañados, "Global charges in Chern-Simons theory and the 2+1 black hole", Phys. Rev. D52 $5816,1996$. arXiv:hep-th/9405171 [hep-th]]

[6] M. Blagojević, Gravitation and gauge symmetries, IOP Publishing, 2002.

[7] M. Bañados and I.A. Reyes, "A short review on Noether's theorems, gauge symmetries and boundary terms", 2016. arXiv:1601.03616 [hep-th]]

[8] C. Troessaert, "Canonical structure of field theories with boundaries and applications to gauge theories", 2013. arXiv:1312.6427 [hep-th]]

[9] G. Arcioni, M. Blau and M. O'Loughlin, "On the boundary dynamics of Chern-Simons gravity “, JHEP 0301, $067,2003$. arXiv:hep-th/0210089 [hep-th]]

[10] A. Gallardo and M. Montesinos, "The boundary field theory induced by the Chern-Simons theory", J. Phys. A44, 135402, 2011 arXiv:1008.4883 [hep-th]].

[11] A.P. Balachandran, G. Bimonte, K.S. Gupta and A. Stern, "Conformal edge currents in Chern-Simons theories", Int. J. Mod. Phys. A7 (1992) 1992. arXiv:hep-th/9110072 [hep-th]]

[12] A.P. Balachandran, G. Bimonte, K.S. Gupta and A. Stern, "The Chern-Simons source as a conformal family and its vertex operators", Int. J. Mod. Phys. A7 5855, 1992. arXiv:hep-th/9201048 [hep-th]]

[13] R. Floreanini, R. Percacci and R. Rajaraman, "Four dimensional current algebra from Chern-Simons theory", Phys. Lett. B231, 119, 1989. 
[14] K.S. Gupta and A. Stern, "4D edge currents from 5D Chern-Simons theory", Nucl. Phys. B442, $157,1995$. arXiv:hep-th/9410216 [hep-th]]

[15] V. Husain and S. Major, "Gravity and BF theory defined in bounded regions", Nucl. Phys. B500, $381,1997$. arXiv:gr-qc/9703043 [gr-qc]]

[16] A.P. Balachandran, G. Bimonte and P. Teotonio-Sobrinho, "Edge states in 4-d and their 3-d groups and fields", Mod. Phys. Lett. A8, 1305, 1993. arXiv:hep-th/9301120 [hep-th]]

[17] M. Bañados, L.J. Garay and M. Henneaux, "The dynamical structure of higher dimensional Chern-Simons theory" Nucl.Phys. B476, 611, 1996. arXiv:hep-th/9605159 [hep-th]]

[18] A. Corichi, I. Rubalcava-García, and T. Vukašinac, "Actions, topological terms and boundaries in first-order gravity: A review", Int. J. Mod. Phys., D25(04):1630011, 2016. arXiv:1604.07764 [gr-qc]]

[19] T. Regge and C. Teitelboim, "Role of surface integrals in the Hamiltonian formulation of general relativity", Annals Phys. 88, 286, 1974.

[20] M. Zabzine, "Hamiltonian systems with boundaries", JHEP 0010 042, 2000. arXiv:hep-th/0005142 [hep-th]]

[21] M. M. Sheikh-Jabbari and A. Shirzad, "Boundary conditions as Dirac constraints", Eur. Phys. J. C19, $383,2001$. arXiv:hep-th/9907055 [hep-th]]

[22] J. M. Romero and J. D. Vergara, "Boundary conditions as constraints", 2002 [arXiv:hep-th/0212035 [hep-th]].

[23] M.J. Gotay, J.M. Nester and G. Hinds, "Presymplectic manifolds and Dirac-Bergmann theory of constraints", J. Math. Phys. 19, 2388, 1978.

[24] J.F. Barbero, J. Prieto and E.J.S. Villaseñor, "Hamiltonian treatment of linear field theories in the presence of boundaries: a geometric approach", Class. Quant. Grav. 31, 045021, 2014. arXiv:1306.5854 [math-ph]]

[25] A. Escalante and L. Carbajal, "Hamiltonian study for Chern-Simons and Pontryagin theories", Annals Phys. 326 323, 2011. arXiv:1107.4023 [math-ph]]

[26] O. Coussaert, M. Henneaux and P. van Driel, "The asymptotic dynamics of three-dimensional Einstein gravity with a negative cosmological constant", Class. Quant. Grav. 12, 2961, 1995. arXiv:gr-qc/9506019 [gr-qc]]

[27] J.M. Maldacena, "The large N Limit of superconformal field theories and supergravity", Adv. Theor. Math. Phys. 2, 231, 1998. arXiv:hep-th/9711200 [hep-th]]

[28] E. Witten, "Anti de Sitter space and holography", Adv. Theor. Math. Phys. 2, 253, 1998. arXiv:hep-th/9802150 [hep-th]]

[29] L. Castellani, "Symmetries in constrained Hamiltonian systems", Annals Phys. 143, 357, 1982.

[30] V. O. Solovev, "Bering's proposal for boundary contribution to the Poisson bracket", J. Math. Phys. 41, 5369, 2000 arXiv:hep-th/9901112 [hep-th]]; V. O. Solovev, "Boundary values as Hamiltonian variables. 1. New Poisson brackets", J. Math. Phys. 34, 5747, 1993 arXiv:hep-th/9305133 [hep-th]]; K. Bering, "Family of boundary Poisson brackets", Phys. Lett. B486, 426, 2000 arXiv:hep-th/9912017[hep-th]].

[31] A. Corichi and T. Vukašinac, "Canonical Hamiltonian analysis of Maxwell-Pontryagin in the presence of boundaries", to be submitted (2019). 